\title{
Kosterlitz-Thouless transition and conductivity fluctuations in Y-Ba-Cu-O thin films
}

\author{
Q. Y. Ying \\ Department of Physics and Astronomy, State University of New York at Buffalo, Buffalo, New York 14260
}

\author{
H. S. Kwok \\ Institute of Superconductivity, State University of New York at Buffalo, Buffalo, New York 14260
}

(Received 2 January 1990)

\begin{abstract}
Kosterlitz-Thouless properties of $c$-axis-oriented high- $T_{c}$ superconducting $\mathrm{YBa}_{2} \mathrm{Cu}_{3} \mathrm{O}_{7-x}$ thin films were examined. The current-voltage characteristics over a wide current range obeyed a power-law relationship. The exponent $\alpha(T)$ showed a "universal jump" at $T_{c}$. The exponential inverse-square-root reduced-temperature dependence of the resistance above $T_{c}$ was consistent with a two-dimensional (2D) behavior of the thin films. Excess conductivity due to thermal fluctuations of the order parameter above the mean-field transition temperature $T_{c(0)}$ was also measured. However, the paraconductivity showed a $1 \mathrm{D}$-like behavior at temperatures close to $T_{c 0}$ and further complications at higher temperatures. This $1 \mathrm{D}$-like behavior could be the result of imperfections of the dimensionality of 2 in the polycrystalline sample.
\end{abstract}

In the last decade, there has been considerable effort to investigate both theoretically and experimentally the Kosterlitz-Thouless (KT) properties ${ }^{1}$ in two-dimensional superconductors, namely, superconducting thin films. ${ }^{2,3}$ In high- $T_{c}$ copper oxide superconductors, there exists an intrinsic two-dimensional (2D) $\mathrm{Cu}-\mathrm{O}$ structure and large anisotropy between the $c$ axis and the $a-b$ plane. This strongly suggests 2D-like behavior for the superconducting transition. Indeed, the KT transition of the high- $T_{c}$ materials has been studied, and confirmation of the 2D behavior has been reported for a variety of systems. ${ }^{4-10}$ Kosterlitz-Thouless properties have been examined in $\mathrm{YBa}_{2} \mathrm{Cu}_{3} \mathrm{O}_{7}$ thin films in the study of mean-field penetration-depth renormalization. ${ }^{4}$ Similar studies were carried out in bulk, single-crystal $\mathrm{YBa}_{2} \mathrm{Cu}_{3} \mathrm{O}_{7}$ and $\mathrm{Bi}_{2} \mathrm{Sr}_{2} \mathrm{CaCu}_{2} \mathrm{O}_{8}$ samples. ${ }^{5-8}$ Also, a phase transition associated with vortex unbinding has been observed in granular $\mathrm{YBa}_{2} \mathrm{Cu}_{3} \mathrm{O}_{7}$ films in optical-response studies. ${ }^{9}$ Most recently, the $\mathrm{KT}$ transition was found in $\mathrm{Tl}_{2} \mathrm{Ba}_{2} \mathrm{CaCu}_{2} \mathrm{O}_{8}$ films. ${ }^{10}$

The dimensionality of the order parameter could also be probed through its thermal fluctuations above the mean-field transition temperature $T_{c 0}$. This is because the excess conductivity depends on the reduced temperature differently according to its dimensionality. ${ }^{11}$ Results previously reported in the literature showed complicated properties of 3D (Refs. 12-14), 2D (Refs. 15-18), and crossover of the dimensionality ${ }^{19}$ in the high $-T_{c}$ materials.

Obviously, the dimensionality of the superconducting transition is important in understanding the physics of these new superconductors. To a large extent, in addition to the crystalline structure, the dimensionality also depends on the nature of the sample, whether it is bulksintered powder, polycrystalline, or single crystalline. For $\mathrm{YBa}_{2} \mathrm{Cu}_{3} \mathrm{O}_{7}, 3 \mathrm{D}$-like conductivity fluctuations ${ }^{13}$ as well as $2 \mathrm{D}$-like behavior ${ }^{15}$ have been reported for granu- lar bulk materials. In single crystals, exact 2D KT behavior was observed in a careful study with the applied current along, as well as perpendicular, to the $\mathrm{Cu}-\mathrm{O}$ planes. ${ }^{6}$ Similar KT behavior was also reported in granular $\mathrm{YBa}_{2} \mathrm{Cu}_{3} \mathrm{O}_{7}$ thin films. ${ }^{9}$ One class of material that is very important in applications is the so-called highquality epitaxial $c$-axis oriented films, produced by laser deposition. ${ }^{20-23}$ These films possess excellent superconducting properties in terms of $T_{c}(>85 \mathrm{~K})$ and critical current $J_{c}\left(>10^{6} \mathrm{~A} / \mathrm{cm}^{2}\right.$ at $\left.77 \mathrm{~K}\right) .^{20,21}$ Generally, these films consist of epitaxial single-crystalline grains of $200-1000 \AA$ in size. ${ }^{22,23}$ The $c$ axis is perpendicular to the film surface, while the $a-b$ orientation is textured but not completely aligned. Even in cases where the $a-b$ direction is aligned, there are still misfit boundaries between the grains. The existence of such a pseudorandom network of nearly perfect crystals would obviously affect the $I-V$ characteristics and possibly the dimensionality of the transition. It is the purpose of this paper to report a study of the KT properties and the excess conductivity above $T_{c 0}$ of such $\mathrm{YBa}_{2} \mathrm{Cu}_{3} \mathrm{O}_{7}$ thin films. It should be emphasized here that these high-quality films are different from the intentionally imperfect granular films. ${ }^{9}$

The samples were made by laser deposition using the 193-nm ArF excimer laser. ${ }^{21}$ These films and the laser deposition method have been carefully studied. ${ }^{21} \mathrm{X}$-ray diffraction peaks as well as transmission electron microscopy lattice images showed $c$-axis orientation perpendicular to the (100) MgO substrate. The film thickness used in this study was $0.12 \mu \mathrm{m}$. The sample was patterned into a strip with use of a direct laser ablation technique ${ }^{21}$ and was $80 \mu \mathrm{m}$ wide by $6.5 \mathrm{~mm}$ long. The standard four-probe method was used for the $I-V$ measurement near zero magnetic field $(0.5 \mathrm{G})$. As will be discussed later, this nonzero magnetic field did not affect deleteriously the $I-V$ measurement. The temperature stability during each measurement was $<0.05 \mathrm{~K}$. For the excess 
conductivity measurement, the bias current was $1 \mu \mathrm{A}$. The uncertainty of the temperature during cooling was $<0.15 \mathrm{~K}$. Special care was taken to avoid sample heating by the applied current.

The Kosterlitz-Thouless transition was associated with the intrinsic vortices in the sample. The binding energy between a vortex-antivortex pair at zero electric current is given by

$$
U(r)=2 E_{c}+q^{2} \ln (r / \xi) \text { for } \xi \ll r \ll L_{s},
$$

where $q=\left(\pi n_{s} \hbar^{2} / 2 m\right)^{1 / 2}$ is the effective charge, $E_{c}$ the vortex core energy, $n_{s}$ the renormalized areal superelectron density, $r$ and $\xi$ the separation of vortex pair and the Ginzburg-Landau coherence length, respectively, and $L_{s}$ is the 2D magnetic penetration depth. Only bound vortex-antivortex pairs exist below certain temperature $T_{c}$, above which vortex unbinding occurs due to the thermal excitation. Under the influence of an electric current, the Lorentz force between the current and the vortices acts as an effective repulsive interaction and would significantly depair the vortex pair if the current exceeds the threshold current

$$
I_{\mathrm{th}}=(2 e / \hbar) k T K\left(l_{m}\right),
$$

where $K\left(l_{m}\right)$ is the Kosterlitz-Thouless reduced stiffness constant and $l_{m}=\ln (w / \xi)$ ( $w$ is the sample width). The flow of the unbounded vortices (flux) under this Lorentz force gives rise to a voltage across the sample and produces a finite dissipation. The current-voltage relationship can be shown to obey the power law ${ }^{2}$

$$
V=I^{\alpha(T)},
$$

where

$$
\alpha(T)=1+q^{2} / 2 k T .
$$

It can be shown that

$$
\begin{array}{ll}
\alpha(T)>3, & T<T_{c}, \\
\alpha(T)=3, & T=T_{c}, \\
\alpha(T)=1, & T>T_{c} \text { for small current } .
\end{array}
$$

The exponent $\alpha(T)$ has a so-called "universal jump" at $T_{c}$, which represents the disappearance of the renormalized superelectron density $n_{s}$. Near this critical region, the exponent should obey the law ${ }^{24}$

$$
\alpha(T)-3=b^{\prime}\left(1-T / T_{c}\right)^{1 / 2},
$$

where $b^{\prime}$ is a constant. The resistance of the sample is related to the effective density of free vortices $n_{f}$ by

$$
\begin{aligned}
R(T) & =2 \pi \xi^{2}(T) R_{n} n_{f} \\
& =a R_{n} \exp \left\{-2\left[b\left(T_{c 0}^{\prime}-T\right) /\left(T-T_{c}\right)\right]^{1 / 2}\right\},
\end{aligned}
$$

where $R_{n}$ is the normal-state resistance, $a, b$, are constants of the order of unity and $T_{c 0}^{\prime}$ is the mean-field Ginzburg-Landau transition temperature. We label the mean-field transition temperature deduced here as $T_{c 0}^{\prime}$. The difference between $T_{c 0}^{\prime}$ and the $T_{c 0}$ obtained from the

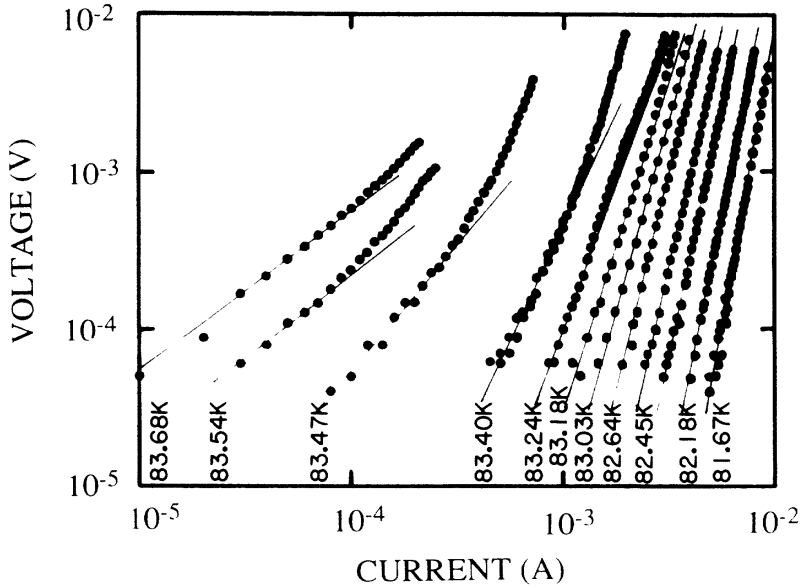

FIG. 1. I-V characteristic of the $\mathrm{Y}-\mathrm{Ba}-\mathrm{Cu}-\mathrm{O}$ thin film near $T_{c}$. A power-law dependence is evident at low currents.

conductivity fluctuations measurement will be discussed.

Figure 1 shows the $I-V$ characteristic of the sample near $T_{c}$. The exponent $\alpha(T)$ was obtained from the slope of the plot in Fig. 1. It is shown in Fig. 2 as a function of $T$. The transition temperature $T_{c}$ was obtained from $\alpha\left(T_{c}\right)=3$ in Fig. 2 and is equal to $83.45 \mathrm{~K}$. In Fig. 3, we show the expected critical behavior of the exponent $\alpha(T)$ using a log-log plot of Eq. (5). It is confirmed that the theoretical slope of 0.5 is followed.

It can also be seen from Fig. 1 that at higher temperatures the $I-V$ relation is linear in the low-current limit, which is the result of thermally dissociated vortexantivortex pairs. At higher current, the "free" vortices contributing to the voltage are mainly from currentinduced depairing where nonlinearity occurs. At lower

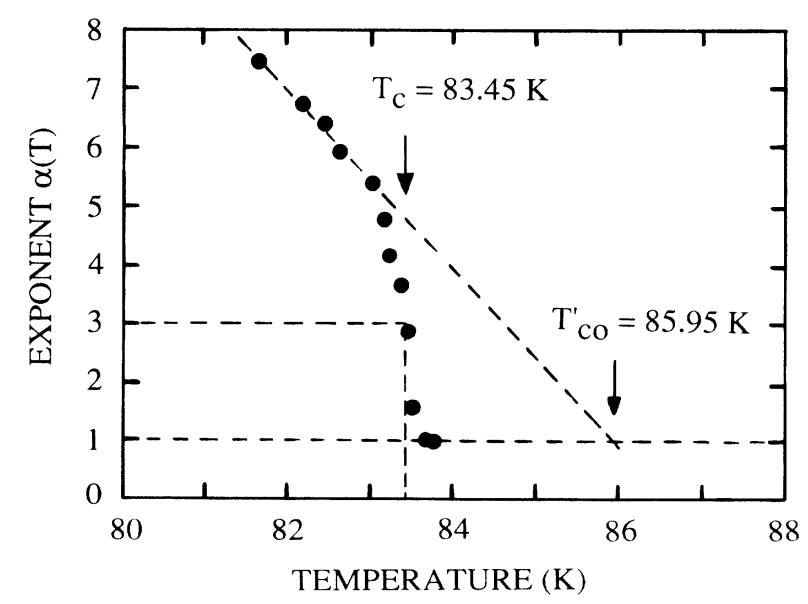

FIG. 2. The exponent as a function of temperature. It shows a "universal jump" at $T_{c}$ from 3 to $1 . T_{c}$ is obtained from $\alpha\left(T_{c}\right)=3$. The corresponding mean-field transition temperature $T_{c 0}^{\prime}=85.95 \mathrm{~K}$. 


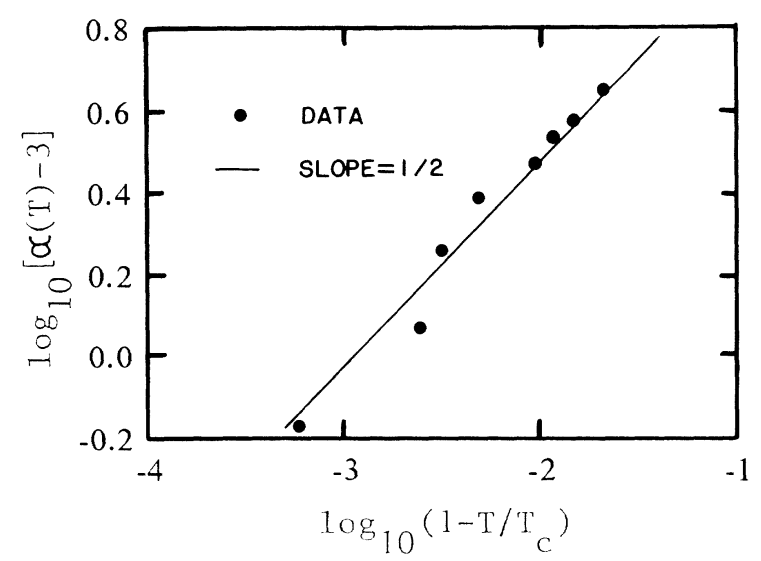

FIG. 3. Critical behavior of $\alpha(T)$ near $T_{c}$. It can be seen that Eq. (5) is obeyed.

temperatures, the free vortices are from the currentinduced depairing.

The earth's flux of $0.5 \mathrm{G}$ could enter the film, since the film thickness $0.12 \mu \mathrm{m}$ was smaller than the magnetic penetration depth of $\sim 0.2 \mu \mathrm{m}$ near $T_{c} \cdot{ }^{4}$ This external magnetic field would have a significant contribution in the $I-V$ measurement if the current is below the threshold value $I_{\text {th }}$ of depairing because those fluxes are free to move if there is no pinning. Should this happen, there would be the change of the slope at the low voltage region in Fig. 1 for all temperatures. ${ }^{25}$ However, our measurement was carried out in the current range well above the threshold value $I_{\text {th }}$, which was estimated from Eq. (2) to be $\sim 3 \mu \mathrm{A}$. In this current range, contributions from thermally dissociated vortices (high temperature) or current-induced vortices (low temperature) dominated. Also notice that the current-induced depinning of the external fluxes was not apparent even at high current density. Therefore, it is concluded that the existence of the 0.5-G external magnetic field does not alter our results.

It should be pointed out that if the external magnetic field and the pinning effect are strong, as the bias current increases, the system will undergo a transition from flux creep to flux flow. In the flux-creep model, below the superconducting transition temperature, the induced voltage $V \sim \sinh \left[I / I_{0}(H, T)\right]$ does not show a power-law dependence. The observed power law, the "universal jump" and critical behavior of $\alpha(T)$, can only be attributed to the vortex-pair unbinding mechanism. Certainly, at higher magnetic fields, flux creep could be dominant, and the $I-V$ curve will change shape accordingly. ${ }^{26,27}$

At temperatures away from $T_{c}$, the renormalized superelectron density $n_{s}$ approaches the Ginzburg-Landau value $\left(n_{s} \sim 1-T / T_{c 0}^{\prime}\right)$. Substituting this into $\alpha(T)$ would give rise to a linear region of $\alpha(T)$ with $a(T)-1$ $\sim\left(1-T / T_{c 0}^{\prime}\right){ }^{24} T_{c 0}^{\prime}(85.96 \mathrm{~K})$ was obtained by extrapolating $\alpha(T)$ to 1 . The dashed line $\alpha_{0}(T)$ in Fig. 2 is the unrenormalized value of $\alpha(T)$. At $T_{c}$, it is related to the dielectric constant due to screening of the vortex-pair interaction [Eq. (1)] by the existence of other vortex pairs with shorter pair separations:

$$
\epsilon\left(T_{c}\right)=\left[\alpha_{0}\left(T_{c}\right)-1\right] / 2 \text {. }
$$

From Fig. 2, we obtain $\epsilon\left(T_{c}\right)=1.9$. This value is close to the values reported for indium oxides ${ }^{28,29}$ and $\mathrm{Hg}-\mathrm{Xe}$ films ${ }^{24,30}$ (1.2-1.8), $\mathrm{Tl}_{2} \mathrm{Ba}_{2} \mathrm{CaCu}_{2} \mathrm{O}_{8}$ films ${ }^{10}$ (2.0). However, it is smaller than that of granular $\mathrm{YBa}_{2} \mathrm{Cu}_{3} \mathrm{O}_{7}$ thin films ${ }^{4}(4.6)$.

It was demonstrated ${ }^{2}$ that the $2 \mathrm{D}$ magnetic penetration depth $L_{s}=2 \lambda_{3 \mathrm{D}}^{2} / d$ is related to $T_{c}$ by $L_{s}$ (in $\mathrm{cm}$ ) $=2.0 / T_{c}$ (in $K$ ), where $\lambda_{3 D}$ is the bulk magnetic penetration depth and $d$ is the film thickness. We can therefore obtain the effective thickness $d_{\text {eff }}$ of each layer, assuming no interaction between the layers. This was estimated to be of or$\operatorname{der} 55 \AA$, by using $\lambda_{3 \mathrm{D}}(T)=(0.15 \mu \mathrm{m})\left(1-T / T_{c 0}^{\prime}\right)^{-1 / 2}$ at $T=83 \mathrm{~K}$. Compared with the film thickness, we can see that it is the intrinsic material properties (large anisotropy) that lead to the quasi-2D behavior. These properties include the short coherence length $\left[\xi_{c}(83 \mathrm{~K}) \sim 16 \mathrm{~A}\right]$ and large anisotropy $\left[\xi_{a b}(83 \mathrm{~K}) \sim 108 \AA\right.$, taking $\xi_{c}(0)=3 \AA$ and $\left.\xi_{a b}(0)=20 \AA\right]$.

The above observations clearly demonstrate the 2D features of the epitaxial $\mathrm{YBa}_{2} \mathrm{Cu}_{3} \mathrm{O}_{7}$ thin film under study. To see the dimensionality information of the superconducting order parameter, excess conductivity above the mean-field transition temperature $T_{c 0}$ was also measured. Generally thermodynamic fluctuations lead to a nonvanishing probability of nonzero superconducting order parameter at $T>T_{c 0}$, which results in an enhanced conductivity. Aslamosov-Larkin (AL) theory ${ }^{31}$ shows that the excess conductivity is given by ${ }^{11}$

$$
\begin{aligned}
& \sigma^{\prime}=\left[e^{2} / 32 \hbar \xi(0)\right] \tau^{-1 / 2}, \quad 3 \mathrm{D}, \\
& \sigma^{\prime}=\left(e^{2} / 16 \hbar d\right) \tau^{-1}, \quad 2 \mathrm{D}, \\
& \sigma^{\prime}=\left[e^{2} / 16 \hbar S \xi(0)^{-1}\right] \tau^{-3 / 2}, \quad 1 \mathrm{D},
\end{aligned}
$$

where $\tau=\left(T-T_{c 0}\right) / T_{c 0}$ is the reduced temperature, and $d$ and $S$ are thickness and cross-sectional area of the sample, respectively. In general, the fitting of Eq. (8) to the experimental results is very sensitive to the value of $T_{c 0}$. To determine the power of the reduced temperature without this parameter $T_{c 0}$, we use the following relationship: ${ }^{18}$

$$
d \sigma^{\prime} / d T \sim\left(\sigma^{\prime}\right)^{(1+\gamma) / \gamma},
$$

where $\sigma^{\prime}=A \tau^{-\gamma}$ and $\gamma$ is equal to $\frac{3}{2}, 1$, and $\frac{1}{2}$ for the 1D, $2 \mathrm{D}$, and $3 \mathrm{D}$ cases, respectively. Therefore a plot of $-d \sigma^{\prime} / d T$ and $\sigma^{\prime}$ will provide the dimensionality without the parameter $T_{c 0}$. This plot of the data is shown in Fig. 4. The excess conductivity was obtained from the difference of measured conductivity and the normal-state conductivity. The normal-state conductivity was extrapolated from the temperature range $190 \mathrm{~K}<T<210 \mathrm{~K}$, where the $R-T$ relation is linear. This temperature range was chosen so as to have $T>2 T_{c 0}$ and to avoid the resistivity anomaly at $240 \mathrm{~K}$. There are three regions in Fig. 4. Region $A(T<84 \mathrm{~K})$ is the region where the measurement was influenced by the existence of the vortex plasma [Eq. (6)] and the plot deviates from a straight line. The 


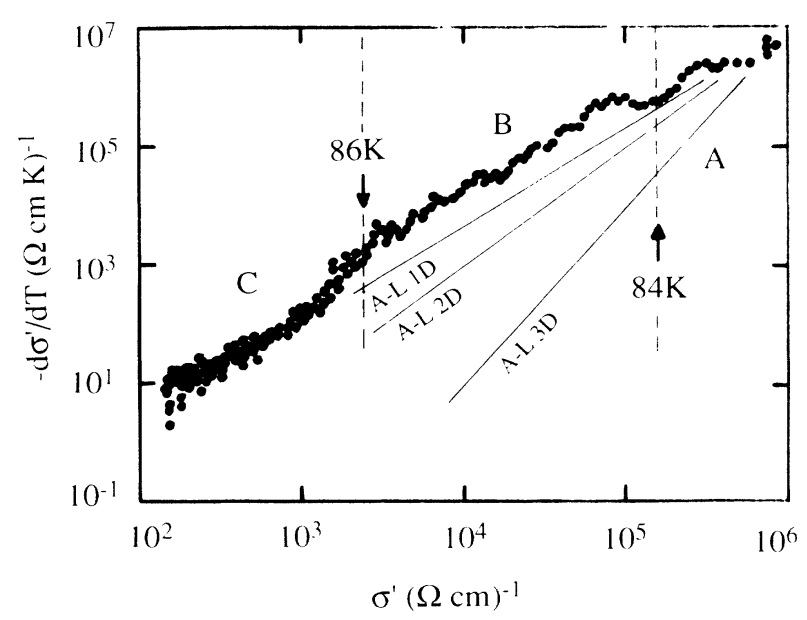

FIG. 4. Plot of Eq. (9) illustrating the 1D behavior of the conductivity fluctuation in temperature region $B(84-86 \mathrm{~K})$. Also plotted are the slopes from Aslamosov-Larkin theory in the $1 \mathrm{D}, 2 \mathrm{D}$, and $3 \mathrm{D}$ cases.

Aslamosov-Larkin (AL) theory applies at $T$ well above this region. Region $B(86 \mathrm{~K}<T<84 \mathrm{~K})$ is the linear region in the plot where the AL theory is valid. It can be seen that the slope in this region corresponds to the $1 \mathrm{D}$ case by comparing it with the theoretical slopes indicated in the plot. This 1D-like behavior occurs at temperature close to $T_{c 0}$. At higher temperature, in region $C$, further complications appear.

Figure 5 shows a plot of Eq. (8c). Since the dimensionality has been determined to be 1 within 84 $\mathrm{K}<T<86 \mathrm{~K}$, we require the slope in Fig. 5 in that temperature range to be $\frac{3}{2}$. It turns out that it is possible only by choosing $T_{c 0}=83.97 \mathrm{~K}$. Different ways of plotting Eq. (8) also confirm that the data in that temperature range are best fitted by the $1 \mathrm{D}$ formula. The deviation at $T>86 \mathrm{~K}$ is similar to other observations reported in the

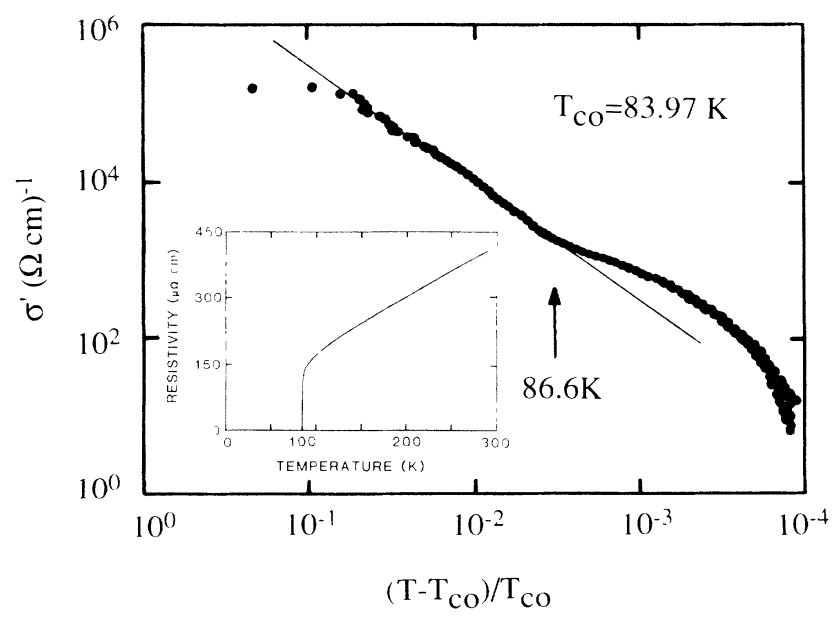

FIG. 5. Plot of Eq. (8c) to determine the mean-field transition temperature $T_{c 0}$. It shows the complications of excess conductivity at $T>86 \mathrm{~K}$ as well. The inset shows the resistivity as a function of temperature. literature. ${ }^{15,17,32}$ Various correction terms (MakiThompson correction) have been suggested, such as a correction due to the sample not being in the dirty limit or due to the existence of paramagnetic impurity or external magnetic field. They will not be elaborated upon here.

Therefore the excess conductivity measurement did not show the $2 \mathrm{D}$ behavior as expected. Instead, it showed a 1D-like behavior between 84 and $86 \mathrm{~K}$. The inconsistency of $T_{c 0}$ and $T_{c 0}^{\prime}$ can also be seen in Fig. 6, which is the plot of Eq. (6b). In Fig. 6, the exponential inversesquare-root reduced temperature dependence was followed at $T<84 \mathrm{~K}\left(\sim T_{c 0}\right)$. However, above this temperature, there is a clear deviation from the 2D behavior represented by Eq. (6b).

To recapitulate, we have observed good agreement with KT transition from the $I-V$ measurements (Fig. 2), which yield a $T_{c 0}$ of $83.45 \mathrm{~K}$ and a Ginzburg-Landau $T_{c 0}^{\prime}$ of $85.95 \mathrm{~K}$. However, from the resistivity dependence below $T_{c 0}^{\prime}$ (Fig. 6) as well as the excess conductivity above $T_{c 0}$ (Fig. 4), a $1 \mathrm{D}$ behavior with a $T_{c 0}$ of $83.97 \mathrm{~K}$ was inferred. This apparent contradiction can be partially explained by noting that $\mathrm{KT}$ transition is associated with $2 \mathrm{D}$ vortices and their interactions. The structure of such $2 \mathrm{D}$ vortices and the range of their interaction have a characteristic length of $2 \lambda_{3 \mathrm{D}}^{2} / d_{\text {eff }}$, which can be estimated to be $\sim 240 \mu \mathrm{m}$ at $T=83 \mathrm{~K}$. Since this value is much larger than the grain size in these laser deposited films, the KT transition behavior would not be influenced by the grain boundaries and the possible percolation structure. This is also why, in granular $\mathrm{In} / \mathrm{InO} \mathrm{O}_{x}$ composite films ${ }^{25}$ and amorphouslike $\mathrm{Hg} / \mathrm{Xe}$ alloy films, ${ }^{24} \mathrm{KT}$ transition was observed.

On the other hand, the resistivity measurement is affected greatly by the grain boundaries and the percolation structure. As one example, the $R-T$ measurement

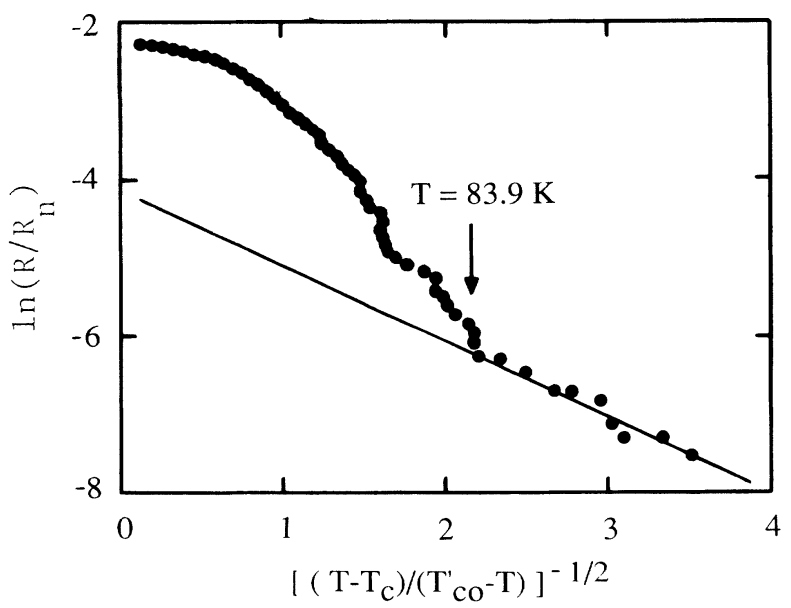

FIG. 6. Log-log plot of Eq. (6b). At $T<84 \mathrm{~K}$. It follows the prediction of Eq. (6b). The deviation at higher temperature is due to setting in of $1 \mathrm{D}$ conductivity fluctuations. In this plot, the mean-field transition temperature is $T_{c 0}^{\prime}=85.95 \mathrm{~K}$ obtained from the KT transition results. The constants $a$ and $b$ are 0.016 and 0.23 , respectively. 
near $T_{c 0}$ is greatly affected by this polycrystallinity. As shown in the inset of Fig. 5, the linear dependence of $R$ versus $T$ did not extrapolate to zero, and the resistivity at $T=100 \mathrm{~K}$ was larger than that of single crystal. In Refs. 15 and 33, it was pointed out that the dimensionality of the "percolation transition" depends on the relative values of the coherence length and the "connectivity length." Similarly, in polycrystalline bulk $\mathrm{Bi}_{2} \mathrm{Sr}_{2} \mathrm{Ca}_{2} \mathrm{Cu}_{3} \mathrm{O}_{x}$, it was observed that the resistivity behavior deviates from the 2D Aslamosov-Larkin theory near $T_{c 0}$ and was attributed to weak links between the grains. ${ }^{34}$ Therefore the grain structure has a stronger effect on resistivity measurements particularly at $T_{c 0}$ than on $I-V$ measurements below $T_{c 0}$.

Recently, Kim and co-workers reported that a $2 \mathrm{D}$ form of the Aslamosov-Larkin theory was obeyed for excess conductivity in Tl-based thin films. ${ }^{10,18}$ It is our experience that $\mathrm{Tl}$ films consist generally of much larger grains than $\mathrm{YBa}_{2} \mathrm{Cu}_{3} \mathrm{O}_{7}$ because of the high annealing temperature. With millimeter size grains, these samples can actually be considered as single crystals. In contrast, the laser evaporated "epitaxial" films deposited at low temperature without annealing are morphologically different, and this probably accounts for the pseudo-1D behavior. Historically, 1D behavior of the excess conductivity has been observed in whiskers, ${ }^{35}$ aluminum microstrips, ${ }^{38,39}$ and tin wires. ${ }^{38}$ We suspect that in the case of epitaxial polycrystalline films, it is possible to have $1 \mathrm{D}$-to-3D behavior depending on the percolation structure.

In summary, we observed the Kosterlitz-Thouless transition in high-quality laser deposited $\mathrm{YBa}_{2} \mathrm{Cu}_{3} \mathrm{O}_{7-x}$ superconducting thin films. The $I-V$ characteristic had the predicted power-law dependence. The exponent clearly showed a "universal jump" at $T_{c}$ and the proper critical behavior near $T_{c}$. However, near the mean-field transition temperature from $\mathrm{KT}$ transition, excess conductivity measurement revealed a $1 \mathrm{D}$ behavior. This percolation transition should be investigated further by varying parameters such as grain size and method of sample preparation.

This research was supported partially by the New York State Institute on Superconductivity and the $\mathrm{Na}$ tional Science Foundation.
${ }^{1}$ J. M. Kosterlitz and D. J. Thouless, J. Phys. C 6, 1181 (1973).

${ }^{2}$ B. I. Halperin and D. R. Nelson, J. Low Temp. Phys. 36, 599 (1979).

${ }^{3}$ For brief review, see for example, A. T. Fiory and F. Hebard, Phys. Rev. B 28, 5075 (1983), and references therein.

${ }^{4}$ A. T. Fiory, A. F. Hebard, P. M. Mankiewich, and R. E. Howard, Phys. Rev. Lett. 61, 1419 (1988).

${ }^{5}$ S. Martin, A. T. Fiory, R. M. Fleming, G. P. Espinosa, and A. S. Cooper, Phys. Rev. Lett. 62, 677 (1989).

${ }^{6}$ N.-C. Yeh and C. C. Tsuei, Phys. Rev. B 39, 9708 (1989).

${ }^{7}$ M. Sugahara, M. Kojima, N. Yoshikawa, T. Akeyoshi, and N. Haneji, Phys. Lett. A 125, 429 (1987).

${ }^{8}$ P. C. E. Stamp, L. Forro, and C. Ayache, Phys. Rev. B 38, 2847 (1988).

${ }^{9}$ J. C. Culbertson, U. Strom, S. A. Wolf, P. Skeath, and W. K. Burns, Phys. Rev. B 39, 12359 (1989).

${ }^{10}$ D. H. Kim and A. M. Goldman, J. H. Kang, and R. T. Kampwirth, Phys. Rev. B 40, 8834 (1989).

${ }^{11}$ For review, see for example, W. J. Skocpol and M. Tinkham, Rep. Prog. Phys. 38, 1409 (1975).

${ }^{12}$ M. A. Dubson, J. J. Calabrese, S. T. Herbert, D. C. Harris, B. R. Patton, and J. C. Garland, in Novel Superconductivity, edited by S. A. Wolf and V. Z. Kresin (Plenum, New York, 1987), p. 981.

${ }^{13}$ P. P. Freitas, C. C. Tsuei, and T. S. Plaskett, Phys. Rev. B 36, 833 (1987).

${ }^{14}$ B. Oh, K. Char, A. D. Kent, M. Natio, M. R. Beasley, T. H. Geballe, R. H. Hammond, A. Kapitulnik, and J. M. Graybeal, Phys. Rev. B 37, 7861 (1988).

${ }^{15}$ M. Ausloos and Ch. Laurent, Phys. Rev. B 37, 611 (1988).

${ }^{16}$ F. Vidal, J. A. Veria, J. Maza, J. J. Ponte, J. Amador, C. Cascales, M. T. Casais, and I. Rasines, Physica C 156, 165 (1988).

${ }^{17}$ J. A. Veria, J. Maza, and F. Vidal, Phys. Lett. A 131, 310 (1988).
${ }^{18}$ D. H. Kim, A. M. Goldman, J. H. Kang, K. E. Gray, and R. T. Kampwirth, Phys. Rev. B 39, 12275 (1989).

${ }^{19}$ N. P. Ong, Z. Z. Wang, S. Hagen, T. W. Jing, and J. Hovarth, Physica C 153-155, 1072 (1988).

${ }^{20}$ A. Inam, M. S. Hegde, X. D. Wu, T. Venkatesan, P. England, P. F. Miceli, E. W. Chase, C. C. Chang, J. M. Tarascon, and J. B. Wachtman, Appl. Phys. Lett. 53, 908 (1988).

${ }^{21}$ J. P. Zheng, Q. Y. Ying, H. S. Kim, P. Bush, R. Barone, D. T. Shaw, and H. S. Kwok, Appl. Phys. Lett. 55, 1044 (1989).

${ }^{22}$ M. G. Norton, L. A. Tietz, S. R. Summerfelt, and C. B. Carter, Appl. Phys. Lett. 55, 2348 (1989).

${ }^{23}$ D. H. Shin, J. Silcox, S. Russek, D. Lathrop, and R. A. Buhrman (unpublished).

${ }^{24}$ A. M. Kadin, K. Epstein, and A. M. Goldman, Phys. Rev. B 27, 6691 (1983).

${ }^{25}$ J. C. Garland and Hu Jong Lee, Phys. Rev. B 36, 3638 (1987).

${ }^{26}$ J. Z. Sun, K. Char, M. R. Hahn, T. H. Geballe, and A. Kapitulnik, Appl. Phys. Lett. 54, 663 (1989).

${ }^{27}$ T. T. M. Palstra, B. Batlogg, R. B. van Dover, L. F. Schneemeyer, and J. V. Waszczak, Appl. Phys. Lett. 54, 763 (1989).

${ }^{28}$ A. F. Hebard and A. T. Fiory, Phys. Rev. Lett. 44, 291 (1980).

${ }^{29}$ A. T. Fiory, A. F. Hebard, and W. I. Glaberson, Phys. Rev. B 28, 5075 (1983).

${ }^{30}$ K. Epstein, A. M. Goldman, and A. M. Kadin, Phys. Rev. Lett. 47, 534 (1981).

${ }^{31}$ L. G. Aslamasov and A. I. Larkin, Fiz. Tverd. Tela (Leningrad) 10, 1004 (1968) [Sov. Phys.-Solid State 10, 875 (1968)].

${ }^{32}$ F. Vidal, J. A. Veira, J. Maza, J. J. Ponte, F. Garcia-Alvarado, E. Moran, and M. A. Alario, Physica C 153-155, 1371 (1988).

${ }^{33}$ M. A. Dubson, S. T. Herbert, J. J. Calabrese, D. C. Harris, B. R. Patton, and J. C. Garland, Phys. Rev. Lett. 60, 1061 (1988). 
${ }^{34}$ W. Schnelle, E. Braun, H. Broicher, H. Weiss, H. Gens, S. Ruppel, M. Galffy, W. Braunisch, A. Waldorf, F. Seidler, and D. Wohlleben, Physica C 161, 123 (1989).

35 J. W. Coor, Jr., M. J. Skove, E. P. Stillwell, and R. S. Thompson, Phys. Lett. 32A, 445 (1970).
${ }^{36}$ G. A. Thomas and R. D. Parks, Phys. Rev. Lett. 27, 1276 (1971).

${ }^{37}$ A. F. Mayadas and R. B. Laibowitz, Phys. Rev. Lett. 28, 156 (1972).

${ }^{38}$ G. E. Possin, Physica 55, 339 (1971). 